\title{
Wheat blast caused by Magnaporthe oryzae pathovar Triticum is efficiently controlled by the plant defence inducer isotianil
}

\author{
Katharina Portz ${ }^{1} \cdot$ Florencia Casanova $^{1} \cdot$ Angelina Jordine $^{1} \cdot$ Stefan Bohnert ${ }^{1} \cdot$ Andreas Mehl $^{2} \cdot$ Daniela Portz $^{2}$. \\ Ulrich Schaffrath ${ }^{1} \mathbb{B}$
}

Received: 26 June 2020 / Accepted: 8 September 2020 / Published online: 21 September 2020

(c) The Author(s) 2020

\begin{abstract}
Wheat blast caused by Magnaporthe oryzae pv. Triticum is an upcoming threat to wheat cultivation worldwide. The disease crossing over to wheat first gained attention in South America, with increasing interest coming from its more recent appearance in the big wheat-growing areas of Asia. The increasing economic relevance of the disease and the lack of genetic resistance in current wheat breeding material, besides fungicide resistance already present in fungal pathogen populations, highlighted the need to evaluate the potential of isotianil as an alternative plant protection measure. Isotianil is already registered in Asia for the protection of rice against M. oryzae, but because the agronomic practices and disease development of blast differ between rice and wheat, the efficacy of isotianil against wheat blast was hard to predict. Testing isotianil in the currently available formulations, applied either as seed treatment or soil drench, resulted in a significant reduction of disease severity. The efficacy was comparably high, on different wheat cultivars and using several fungal isolates with different degrees of virulence. Microscopic analyses revealed that isotianil treatment can prevent invasive growth of the pathogen. No phytotoxicity from isotianil treatment was observed on wheat plants. Importantly, isotianil not only protects wheat plants at the seedling stage but also on spikes thereby preventing losses due to this most severe disease syndrome. In summary, the results showed the high potential of isotianil to protect against wheat blast.
\end{abstract}

Keywords Wheat blast $\cdot$ Plant defence inducer $\cdot$ Spike infection $\cdot$ Isotianil

\section{Introduction}

Soon after humans began to cultivate plants, devastating plant pathogens emerged, hanging over the heads of farmers like the sword of Damocles. While mankind has striven for a long time to overcome these threats, e.g. thanks to the builder of the first green revolution, Norman Borlaug, who successfully fought against the wheat stem rust (Rajaram 2011), the challenge remains, as diseases evolve and

Electronic supplementary material The online version of this article (https://doi.org/10.1007/s41348-020-00378-y) contains supplementary material, which is available to authorized users.

Ulrich Schaffrath

schaffrath@bio3.rwth-aachen.de

1 Department of Plant Physiology, RWTH Aachen University, 52056 Aachen, Germany

2 Bayer AG, Division Crop Science, 40789 Monheim, Germany adapt; this is especially true in the case of novel races with enhanced virulence, such as the so-called warrior race of yellow rust (Puccinia stiiformis) or race Ug99 of stem rust (Puccinia graminis f.sp. tritici, Pgt) (Ellis et al. 2014). Additionally, pathogens can re-emerge in areas where they were thought to be eradicated, e.g. stem rust (Pgt) having an unexpected outbreak in Germany during 2013 (Olivera Firpo et al. 2017) and appearing in the UK after being absent for almost six decades (Saunders et al. 2019).

A particular example adding to the above-mentioned scenarios is the occurrence of wheat blast, a disease caused by a specific lineage of the Magnaporthe species complex (Gladieux et al. 2018). This group is best known because of Magnaporthe oryzae, a pathogen of rice causing dramatic yield losses of about $\$ 66$ billion annually, equivalent to the amount needed to feed 60 million people (Pennisi 2010). Wheat blast was first observed in South America in 1985 and spread throughout the whole continent reaching Bolivia in 1996 and Argentina in 2012 (Cruz and Valent 2017). Tosa and co-workers provided experimental proof 
that losses of avirulence genes, which previously prevented wheat colonization, together with the widespread use of a novel, high yield wheat cultivar which unintendedly lacked a particular resistance gene, were causal for the host jump of a Lolium-infecting isolate of the Magnaporthe species complex to wheat (Inoue et al. 2017). Until recently, the disease was absent from the big wheat-growing countries in North America, Europe and Asia, but the situation changed dramatically in 2016 and 2017 when it appeared in Bangladesh and India, respectively, most likely via seed imports from South America (Islam et al. 2016). After the disease was reported in Asia, both public and academic attention was raised (Sadat and Choi 2017). An outcome of this situation is the recent debate about the correct naming of the fungus causing wheat blast. While a group headed by Bruce McDonald favours a scenario in which the wheat blast fungus forms a novel species with the name Pyricularia graminis-tritici (Castroagudín et al. 2016; Ceresini et al. 2019), a large international consortium led by Barbara Valent, Nick Talbot and Yukio Tosa, all renowned experts in the field of rice blast disease strongly recommend to treat the wheat blast fungus as a recent tribe (pathotype) of the species Magnaporthe oryzae (asexual state: Pyricularia oryzae) (Zhang et al. 2016; Cruz and Valent 2017; Valent et al. 2019). For this study, we will refer to the pathogen as M. oryzae pathotype Triticum (MoT) (Ceresini et al. 2019; Valent et al. 2019). It is worth noting that while there is an academic discussion regarding nomenclature, there are also far-reaching and very serious practical implications, e.g. on international quarantine regulations, which should not be underestimated.

At farmer level, the economic consequence of infection can also be catastrophic; under favourable climatic conditions, the disease can cause up to $100 \%$ yield losses not least because grains from infected plants are often deformed or shriveled (Cruz and Valent 2017). The latter happens because the pathogen is not only able to infect leaves but also spikes. Most severely, spike infection may also affect the rachis resulting in obstruction of regular grain filling. A low negative correlation between seedling resistance on leaves and adult plant resistance on spikes was reported (Martínez et al. 2019). As mentioned above, the disease can be transmitted by infected seeds, impacting global quarantine measures. Of course, the best way to avoid accidental transmission of the disease is by preventing infection. Because cultivar resistance against wheat blast was not systematically explored in the past, disease control has generally relied upon application of foliar fungicides. Here, the widespread use of strobilurin fungicides already resulted in fungicide resistance present in pathogen populations, e.g. in Brazil (Castroagudin et al. 2015). Moreover, the intensive application of fungicides to control other diseases inadvertently was selected for $M o T$ isolates with resistance against quinone outside inhibitors (QoIs, e.g. strobilurins) and demethylation inhibitors (DMIs, e.g. azoles) (Ceresini et al. 2019). Therefore, new, alternative approaches are needed to control this disease. In the light of this necessity, we evaluated the efficacy of isotianil, a plant protection agent developed jointly by Bayer CropScience and Sumitomo Chemical Co., Ltd., for the control of rice blast disease and commercially launched during 2010 in Japan and Korea. Although approved only for protection of rice against rice blast or bacterial leaf blight, the disease control spectrum is much broader and covers typical biotrophic bacterial and fungal pathogens but no pathogens with a necrotrophic lifestyle (Toquin et al. 2012). Because isotianil has no direct antimicrobial effect but instead activates typical plant defence reactions, it has been listed as a plant defence inducer or PDI (Toquin et al. 2012; Bektas and Eulgem 2015; Jeschke 2016). This group of compounds triggers the plant immune system and leads to the phenomenon of acquired resistance (Conrath 2009). Gene expression profiling in rice pointed to the salicylic acid (SA) pathway as essential for the mode of action of isotianil because transcripts related to genes involved in SA catabolism and SA signal transduction significantly accumulated after treatment with the chemical and prior to any pathogen challenge (Toquin et al. 2012). Here, we report on a study in which the capacity of isotianil as a measure against wheat blast was evaluated. Different application methods were tested for efficacy on both leaves and spikes, on various combinations of wheat cultivar susceptibility and isolate virulence.

\section{Materials and methods}

\section{Plant material and fungal isolates}

Seeds of wheat plants were germinated on wet filter paper for $24 \mathrm{~h}$ and then transferred to standard soil (type ED73, Balster Einheitserdewerk GmbH, Froendenberg, Germany). Seedlings were kept in a growth chamber with $16 \mathrm{~h}$ light(200-250 $\mu \mathrm{mol} \mathrm{s}^{-1} \mathrm{~m}^{-2}$ ) and $8 \mathrm{~h}$ dark-rhythm at $18{ }^{\circ} \mathrm{C}$ and $65 \%$ relative humidity. The cultivar $(c v$.) 'Little Club' was obtained from seed stocks in the institute while $c v$. Apogee was received from Eckhard Koch, JKI Darmstadt, Germany. Apogee is a wheat cultivar with a very short life cycle, flowering after 25 days which makes it an ideal choice to study diseases on spikes under laboratory conditions ( $\mathrm{Li}$ et al. 2017; Wunderle et al. 2012).

Fungal isolates were placed on nylon membrane at $-80{ }^{\circ} \mathrm{C}$ for long-term storage. Isolates for current use were cultivated on oatmeal agar $\left(20 \mathrm{~g} \mathrm{l}^{-1}\right.$ agar, $2 \mathrm{~g} \mathrm{l}^{-1}$ yeast extract, $10 \mathrm{~g} \mathrm{l}^{-1}$ starch, $30 \mathrm{~g} \mathrm{l}^{-1}$ oat flakes) at $23^{\circ} \mathrm{C}$ in the dark. Sporulation was induced by subjecting fungal cultures to a $16 \mathrm{~h} / 8 \mathrm{~h} \mathrm{light/dark} \mathrm{regime} \mathrm{under} \mathrm{black} \mathrm{light} \mathrm{at} 22{ }^{\circ} \mathrm{C}$. 
The MoT isolates Br116.5 and BR32 were obtained from Yukio Tosa, Kobe University, Japan, and Didier Tharreau, CIRAD Montpellier, France, respectively. The isolates AR06 and AR33 used in this study were obtained from a collection of MoT isolates maintained at CIDEFI (Centro de Investigaciones de Fitopatología) which belongs to the Facultad de Ciencias Agrarias y Forestales-Universidad Nacional de La Plata, Buenos Aires, Argentina.

\section{Plant inoculation}

Preparation of inoculum was according to the method of Delventhal et al. (2014). In brief, conidia were harvested from plates 2 weeks after sub-cultivation under black light by rinsing the plates with water and filtering through gauze. Concentration of conidia was counted using a Thoma chamber and adjusted to a final concentration of 250,000 conidiospores $\mathrm{ml}^{-1}$ in spraying solution $\left(2 \mathrm{~g} \mathrm{l}^{-1}\right.$ gelatin, $1 \mathrm{ml}^{-1}$ Tween). For inoculation at the seedling stage, the conidial suspension was sprayed onto leaves and plants, which were then kept in the dark at $24-26^{\circ} \mathrm{C}$ and $100 \%$ relative humidity for $24 \mathrm{~h}$. Thereafter, plants were transferred to a growth chamber, still covered with a plastic hood and kept under the conditions previously described. Spike inoculation was carried out 2 days after full emergence of the spike. Each spike was separately inoculated with one $\mathrm{ml}$ of spore suspension and immediately covered with a pre-wetted plastic bag to maintain high humidity. Plants were placed in the growth chamber, with bags remaining in place, for 3 days before removal. Preparation of leaf samples for microscopy and assignment of cellular interaction phenotypes to particular categories is described in (Delventhal et al. 2014).

\section{Treatment with isotianil}

Isotianil was applied as either the seed treatment formulation FS 200 or the drench formulation SC 200. For seed treatment, $5 \mathrm{~g}$ of wheat seeds were placed in a Falcon tube and covered with $50 \mu \mathrm{l}$ of a 1:4 dilution of FS 200 in distilled water. This amount of isotianil equates to $50 \mathrm{~g}$ a.i. (active ingredient) per dt (decitonnes). Seeds were mixed with the diluted formulation until completely covered. Drench application (13 $\mathrm{mg}$ or $26 \mathrm{mg}$ a.i. per pot) was performed by soaking each pot $(350 \mathrm{ml}$ soil volume) with $40 \mathrm{ml}$ of isotianil suspension ( $57 \mu \mathrm{l}$ or $114 \mu \mathrm{l}$ isotianil SC 200 diluted in $40 \mathrm{ml}$ distilled water). For this procedure, each pot was placed on an open Petri dish before soaking and kept separately until complete absorbance of the solution. For spray application, an isotianil suspension was prepared by adding $100 \mu \mathrm{l}$ of isotianil SC 200 to $60 \mathrm{ml}$ distilled water which equals $200 \mathrm{~g}$ a.i. per ha (hectare). The solution was sprayed until 'run-off', i.e. no more could be retained by the plant surface.

\section{Quantitative disease scoring}

Wheat leaves with clearly visible disease symptoms were laid flat on water agar plates $(1 \%(\mathrm{w} / \mathrm{v})$ agar in distilled water). Pictures of ten leaves per treatment were photographed under indirect light with a camera avoiding light reflections. Quantification of the diseased leaf area was made with the APS software tool Assess 2.0. At first, the threshold was roughly set using the Automatic Panel and thereafter adjusted with the Manual Panel to enable a clear differentiation between healthy and diseased leaf areas. The same settings were applied to all samples from each experiment. Values of the diseased leaf area are given as percentages of the total leaf area.

\section{Quantitative real-time PCR}

Total RNA was extracted from secondary wheat leaves (one sample consisting of two leaves, treated as described above) using the citric acid protocol described by Mogga et al. (2016); the material was suspended in $600 \mu \mathrm{l}$ cell lysis buffer, and $200 \mu \mathrm{l}$ precipitation buffer was added to separate proteins and DNA from the RNA. After that, the RNA was precipitated from the supernatant with isopropanol, washed in $70 \%$ ethanol and dissolved in double-distilled water. After digestion of DNA with DNase I (Thermo Fisher Scientific Inc., Germany), cDNA synthesis was performed for each RNA sample (1 $\mu$ g RNA) using reverse transcription (RevertAid Reverse Transcriptase, Thermo Fisher Scientific Inc., Germany) with HindAnchorT-primer. RT-qPCR was done using iTaq Universal SYBR Green Supermix (Bio-Rad Laboratories Inc. USA), the CFX384 Touch Real-Time PCR Detection System (Bio-Rad Laboratories Inc. USA) and the following gene-specific primers: WCI-2_F 5'-ATCACGAGC CAGCTGCAAA-3', WCI-2_R 5'-GCCTTTTTCGCCTTG ACATC-3' (Sardesai et al. 2005), EF1 $\alpha \_F$ 5'-ATGATTCCC ACCAAGCCCAT-3', EF1 $\alpha \_R$ 5'-ACACCAACAGCCACA GTTTGC-3' (McGrann et al. 2015). Reactions were heated to $95{ }^{\circ} \mathrm{C}$ for $3 \mathrm{~min}$, followed by 40 cycles of $95^{\circ} \mathrm{C}$ for $10 \mathrm{~s}$ and $59{ }^{\circ} \mathrm{C}$ for $30 \mathrm{~s}$. After the final PCR cycle, the reactions were terminated by heating up to $95{ }^{\circ} \mathrm{C}$ for $10 \mathrm{~s}$ and a melting curve analysis was carried out for each sample. Transcript abundance of the target gene 'WHEAT CHEMICALLY INDUCED 2' (WCI-2) was calculated relative to the transcript abundance of the reference gene 'ELONGATION FACTOR 1 $\alpha^{\prime}(E F 1 \alpha)\left(2^{\text {(Ct(reference)-Ct(target) })}\right)$ (Livak and Schmittgen 2001). 


\section{Results}

The wheat blast pathogen $M o T$ is able to cause disease symptoms on leaves and ears of infected wheat plants. A causal relationship between sporulation of the fungus on leaves and the later occurrence of ear infections is still a matter of debate, and accordingly, it is questioned whether screening for resistance against MoT on wheat leaves provides reliable answers for resistance expressed on ears. In response to these unsolved questions, we decided to test the effectiveness of isotianil against wheat blast on both plant organs, i.e. leaves and ears. As known from other pathosystems, the efficacy of isotianil can vary depending on the application method, and therefore, seed dressing, soil drenching and foliar spraying were all included.

\section{Isotianil effectively protects wheat plants at the seedling stage against $M o T$}

Seed treatment was made prior to sowing using two different concentrations of isotianil which equals 10 or $50 \mathrm{~g}$ a.i. per dt seed as suggested by the supplier (IST 10 and IST 50, Fig. 1 and Online Resource 1). Plants derived from these seeds and plants from untreated seeds which served as control (UTC) were inoculated with fungal conidia 17 days after sowing, on the third fully expanded leaf. Disease severity was scored 1 week after inoculation by quantification of the diseased leaf area using digital imaging. Both the area and length of disease symptoms caused by MoT on inoculated leaves of isotianil-treated plants were significantly reduced compared to untreated control plants (Online Resource 1a). Evaluation of the efficacy of seed treatment with isotianil was carried out over four independent experiments. To enable comparability between replicates, the UTC value for diseased leaf area was set in each experiment to $100 \%$ (Online Resource 1a). A significant reduction of diseased leaf area on isotianil-treated plants could be shown in all biological replicates. This reduction was on average $70 \%$ and $82 \%$ for a treatment with 10 and $50 \mathrm{~g}$ a.i. per dt, respectively. Importantly, the degree of protection was equally good, regardless of the disease severity on control plants (UTC) which varied between experiments in a range from 1 to $40 \%$ (Online Resource $1 \mathrm{~b}$ ). The results from quadruple experiments were calculated as relative reduction in diseased leaf area in comparison with UTC and then expressed as mean and standard error in Fig. 1. In a similar way, drench and spray treatments were evaluated in quadruple experiments using different concentrations of isotianil and altering the time span between treatment and inoculation. All results are given for individual experiments (Online Resources 2 and 3) and summarized in Fig. 1. From these results, it was obvious that drench treatment performed best in protecting wheat against MoT; drenching with solutions of 13 or $26 \mathrm{mg}$ a.i. per pot (recommendation of supplier) at 5 or 7 days prior to inoculation resulted in an almost $100 \%$ protection rate

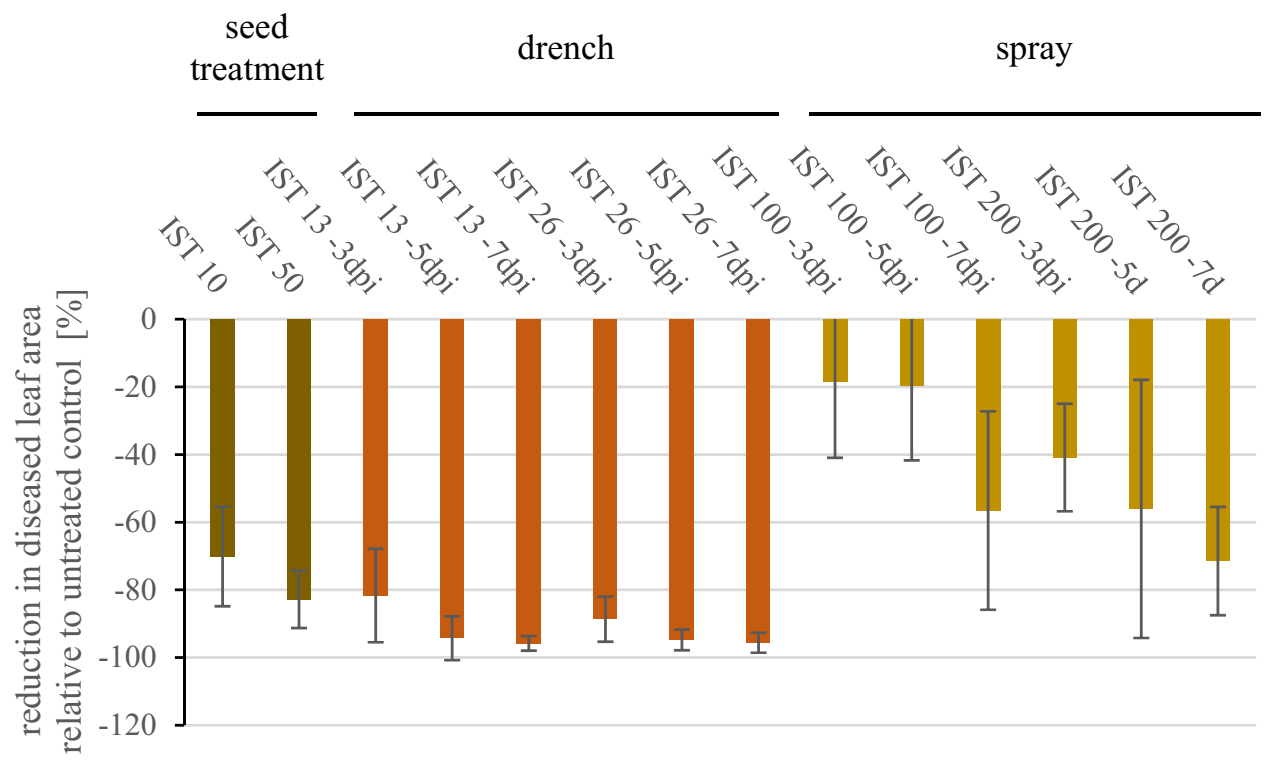

Fig. 1 Effectiveness of isotianil (IST) in controlling wheat blast using different methods of application. Isotianil was applied to wheat plants $c v$. Little Club either by seed, drench or spray treatment at different concentrations and at varying time points prior to inoculation with the virulent MoT isolate BR32. Disease scoring was done on the third leaf 1 week after inoculation by taking photographs of leaves from 10 plants. Diseased leaf area was determined on photographs and quantitatively evaluated with the software APS Assess 2.0. Values shown are the mean and standard error of four independent biological replicates and represent the reduction of diseased leaf area relative to untreated control plants. Details of individual experiments are given in Online Resources 1-3 
(Fig. 1). Merely applying $13 \mathrm{mg}$ a.i. per pot at 3 days prior to inoculation showed a lower efficacy of $80 \%$ reduction of diseased leaf area relative to respective control plants (UTC). This was quite similar to the seed treatment with $50 \mathrm{~g}$ a.i. per dt. Noticeably, spray treatments with different concentrations of isotianil at 3, 5 or 7 days prior to inoculation gave more variable results and a generally lower degree of protection with $70 \%$ reduced infected leaf area being best after treatment with $200 \mathrm{~g}$ a.i. per hectare at 7 days before inoculation.

\section{Aggressiveness of MoT isolates did not influence protection by isotianil}

Experiments shown above were obtained with the wheat cultivar, 'Little Club' and MoT isolate BR32. In these tests, although the disease severity on untreated plants varied between biological replicates, this did not influence the efficacy of isotianil treatments (Online Resources 1-3). To broaden the base for this observation, the set-up was extended by including an additional wheat cultivar, i.e. Apogee, and three additional isolates of MoT (Online Resource 4). Macroscopic evaluation of disease severity revealed the highest aggressiveness for the Argentinian isolates AR06 and AR33 on both wheat cultivars. In contrast, isolates BR32 and BR116.5 showed a lower infection rate on $c v$. Little Club compared with $c v$. Apogee (Online Resource 4). Because drench application was shown to be most effective in previous experiments (Fig. 1), this technique was used to apply isotianil, at a concentration of $26 \mathrm{mg}$ a.i. per pot, 5 days prior to inoculation of the second leaf. In all cases, treated plants were clearly less severely infected following artificial inoculation MoT (all isolates on both wheat cultivars). Quantitative assessment of this experiment was carried out in an analogous way to the results already presented in Fig. 1. This evaluation substantiated the macroscopic observations by confirming that both wheat cultivars were similarly highly susceptible to the Argentinian MoT isolates AR06 and AR33, with leaves showing 40-45\% diseased area (Fig. 2a). By contrast, leaves of wheat cultivar Apogee were less severely affected by the Brazilian isolates BR32 and BR116.5 at only 5 and $10 \%$ of diseased leaf area, respectively, with $c v$. Little Club being even less susceptible at approximately 5\% diseased leaf area from either isolate (Fig. 2a). Remarkably, the degree of protection after drench application of isotianil was comparable high (approx. 75-90\%) for both wheat cultivars, when attacked by the most aggressive isolates AR06 and AR33 (Fig. 2b). Nonetheless, even on plants which are only slightly infected in this particular experiment, i.e. Apogee and Little Club after inoculation with BR32, a $60 \%$ reduction in diseased leaf was recorded.

Extensive use of fungicides may cause shifts in pathogen populations towards isolates showing fungicide resistance

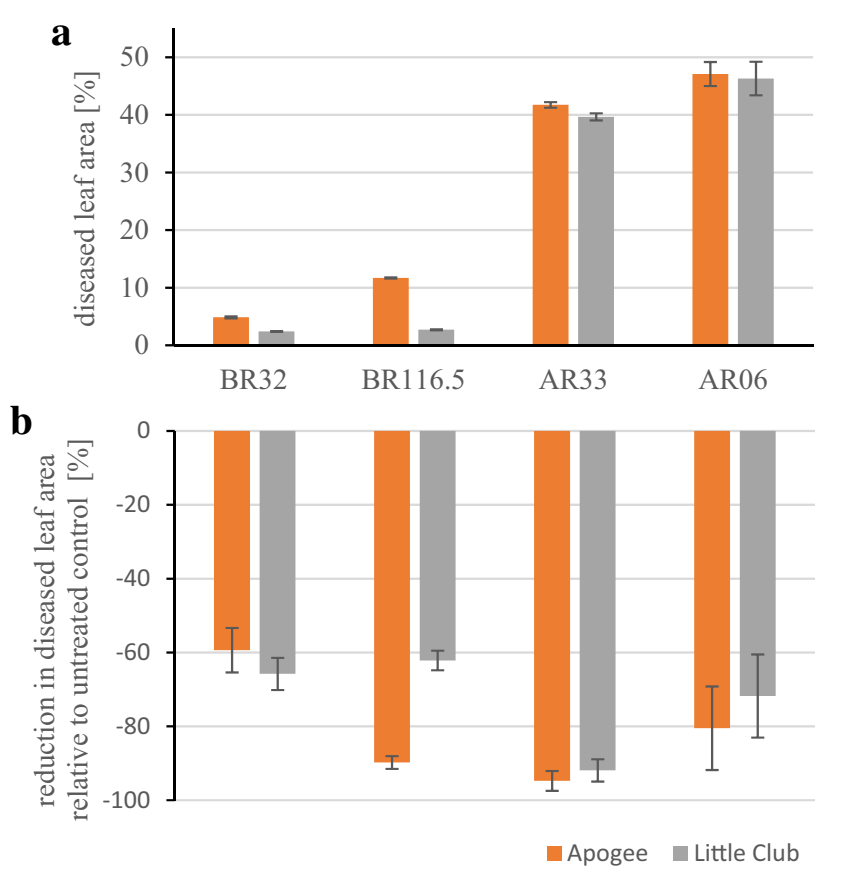

Fig. 2 Degree of susceptibility of wheat cultivars against different isolates of MoT. The two wheat cultivars Apogee and Little club were tested for their response towards different isolates of the wheat blast fungus. The isolates originated either from Brazil (BR32 and BR116.5) or Argentina (AR33 and AR06). Wheat plants were grown until full emergence of the second leaf, and then, ten plants were treated with isotianil at $26 \mathrm{mg}$ a.i./pot by drench, whereas the other ten plants remained untreated. After additional 5 days, all plants were inoculated with conidia of isolates as indicated. Disease scoring was made on the third leaf 1 week after inoculation by taking photographs of ten leaves. Diseased leaf areas were determined on photographs and quantitatively evaluated with the software APS Assess 2.0. Values shown are the mean and standard error. a Comparison of susceptibility for both wheat cultivars to $M o T$ isolates. b Reduction of diseased leaf area on plants treated with isotianil relative to untreated plants. Pictures of disease symptoms on treated and untreated plants are shown in Online Resource 4

which eventually is correlated with a fitness penalty (Hawkins and Fraaije 2018). It is well known that MoT populations in Brazil display resistance to strobilurin fungicides (so-called quinone outside inhibitors, QoI) by non-synonymous nucleotide substitutions in the cytochrome $b$ gene that prevents fungicide binding. While the G143A mutation, occurring at a frequency of $36 \%$, provides complete fungicide resistance, the F129L mutation confers moderate resistance (Castroagudin et al. 2015). To check for the possibility that the Brazilian isolates BR32 and BR116.5 contain these mutations and therefore are less aggressive on their host, as similarly reported for $M$. oryzae on perennial ryegrass (Ma and Uddin 2009), a PCR-based analysis was performed (Online Resource 5). The results conclusively showed that neither the G143A nor the F129L mutations were present in isolates used in this study. 


\section{Microscopic analysis revealed that isotianil prevents invasive growth of MoT}

To determine the mode of action of isotianil on pathogen colonization, a histological analysis was performed. As reported in previous studies, plant-pathogen interaction sites, i.e. plant cells attacked by the fungus, were inspected and grouped into four different categories which are associated with plant defence or successful pathogen development (Online Resource 6) (Delventhal et al. 2014). All microscopic samples were firstly screened under bright field microscopy on located appressoria, and then, the switch to epi-fluorescent light allowed the determination of auto-fluorescence of the plant cell at the infection site. The deposition of auto-fluorescent material beneath an appressorium can be correlated with a halt of fungal invasion (Jarosch et al. 2003, 2005). In contrast, deposition of auto-fluorescent material associated with collapsed mesophyll cells is a sign of successful pathogen progress from epidermal into mesophyll tissue and is presumably associated with transition from biotrophic to necrotrophic lifestyle. Quantitative assessment of these categories revealed that at 72 and $96 \mathrm{hpi}$, the frequency of infection sites grouped into the fourth category (collapsed mesophyll cells) increased from 5 to $15 \%$ in plants not treated with isotianil (Fig. 3, UTC) which is in accordance

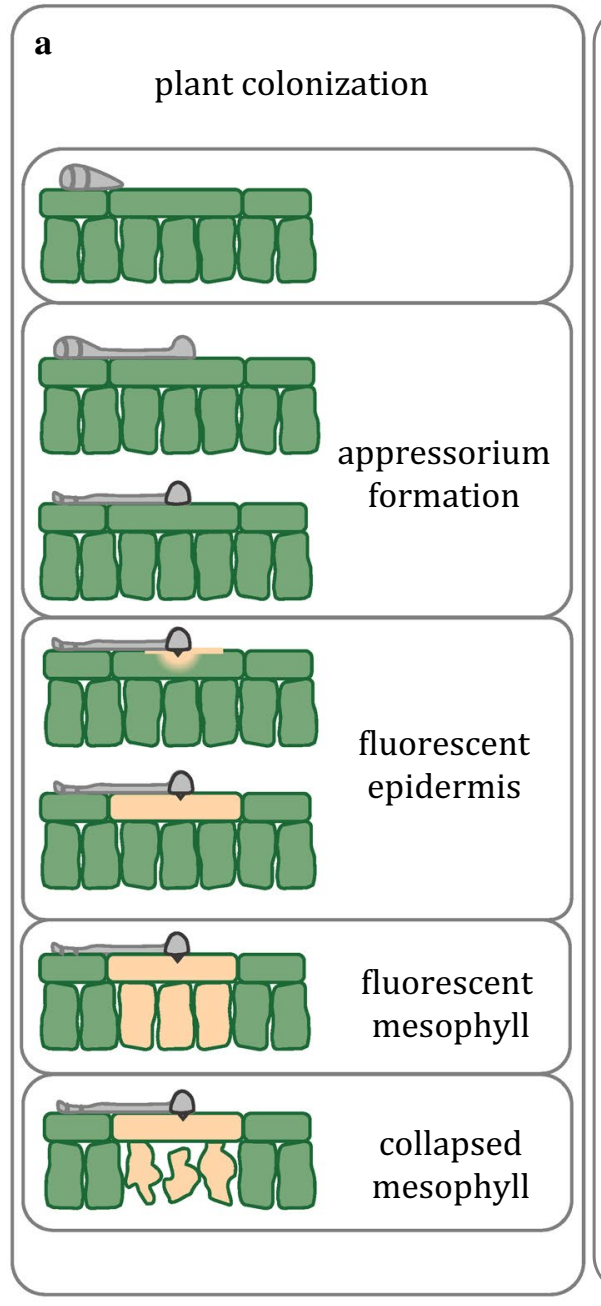

Fig. 3 Quantitative microscopy of wheat plants treated with isotianil and inoculated with $M o T$. The primary leaves of 7-day-old wheat seedlings ( $c v$. Little Club) were drenched with isotianil at $13 \mathrm{mg}$ a.i./ pot. Five days after treatment, secondary leaves were inoculated with the virulent MoT isolate BR32. Inoculated leaves were harvested at $48 \mathrm{hpi}, 72 \mathrm{hpi}$ and $96 \mathrm{hpi}$ and analysed by bright field and epi-fluorescence microscopy after bleaching. a Schematic presentation of the fungal infection process and categories formed in association with

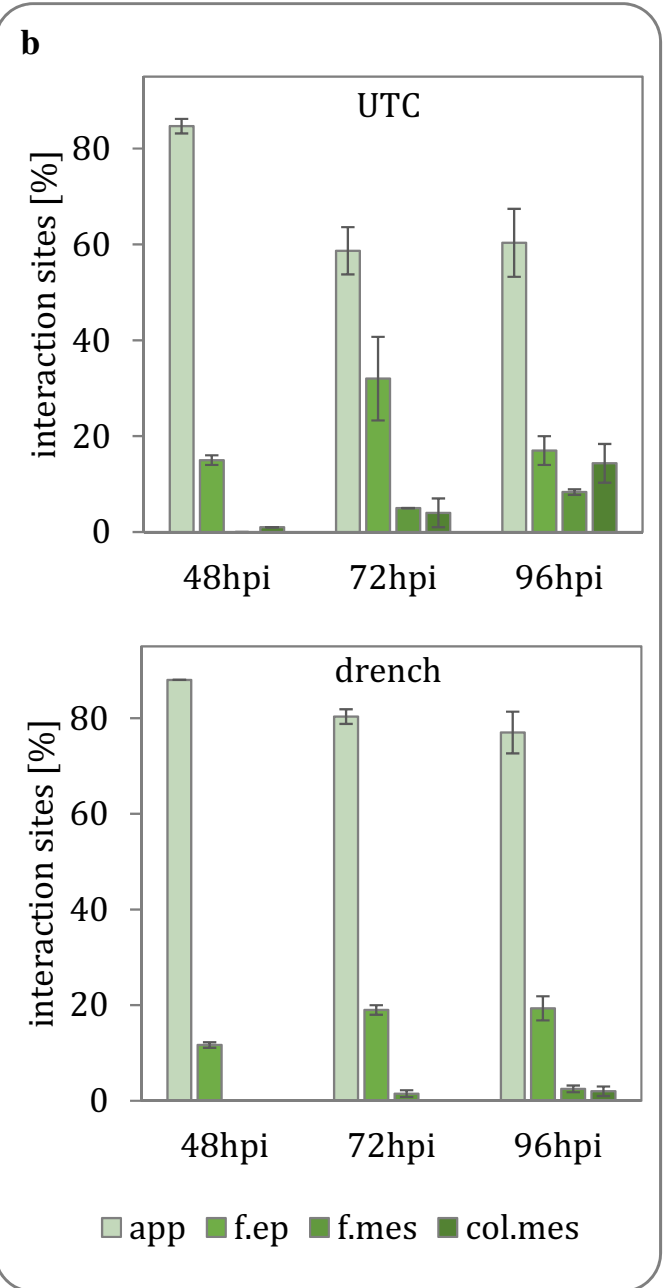

plant responses. b The diagram shows the frequency of these categories per leaf for plants not treated with isotianil (UTC) or plants drenched with the compound (drench) harvested at different time points after inoculation. Values shown represent the mean \pm standard deviation of three leaves consisting of 100 evaluated interaction sites per leaf. Results shown are from a single experiment. hpi, hours postinoculation; app, appressorium; col.mes, collapsed mesophyll; f.ep, fluorescent epidermis; f.mes, fluorescent mesophyll 
with successful infection of MoT isolate BR32 as shown in Fig. 2. By contrast, the frequency of interaction sites grouped into this category was around 1-2\% at 72 and 96 hpi for plants treated with isotianil. This reveals that the pathogen was unable to progress from an initially attacked epidermal cell into the mesophyll, and consequently, pathogen invasion was stopped.

\section{Drench treatment with isotianil at stage of flag leaf development reduces spike infection}

The efficacy of isotianil in protecting wheat against $M o T$ at the seedling stage has been presented above. However, the pathogen is more aggressive in its infection of the spikes (ears). Experiments were therefore also performed to evaluate the efficacy of seed, drench and spray application in this respect. All experiments with spike infections were carried out with $c v$. Apogee because of the exceptionally short time needed for this cultivar to reach 'heading' (Strugala et al. 2015). While seed treatment with isotianil did not show any degree of protection, spray and especially drench application at the stage of fully emerged flag leaves gave remarkable reductions in the number of spikelets showing bleaching due to MoT infection (Fig. 4). The highest degree of protection was achieved by drenching. Inspection of seeds after maturation substantiated this observation (Online Resource 7). According to their size and shape, seeds were categorized either as healthy or shriveled. Control plants neither treated with isotianil nor inoculated with MoT had on average 30 healthy seeds per spike. After inoculation, the proportion of healthy seeds decreased to $48 \%$ and $15 \%$ in two different experiments, while the average number of seeds per spike remained the same (Online Resource 7a and 7b). Consistent with the macroscopic scoring of infected spikes, drench treatment at the flag leaf stage turned out to provide the highest degree of protection with $79 \%$ of healthy seeds per spike (Online Resource 7a). Even more efficient, however, was a combined treatment with two applications of isotianil at first by seed treatment followed by a drench application at the stage of flag leaf expansion. In this case, $90 \%$ of healthy seeds could be recovered from inoculated spikes.

\section{Isotianil treatment triggers expression of the wheat lipoxygenase gene $\mathrm{WCI}-2$}

Plant defence inducers have been intensively studied as protective measure against plant diseases. In the early days, progress in our understanding of the underlying molecular processes was made by correlating transcript abundances with the onset of resistance. At that time, a number of new genes

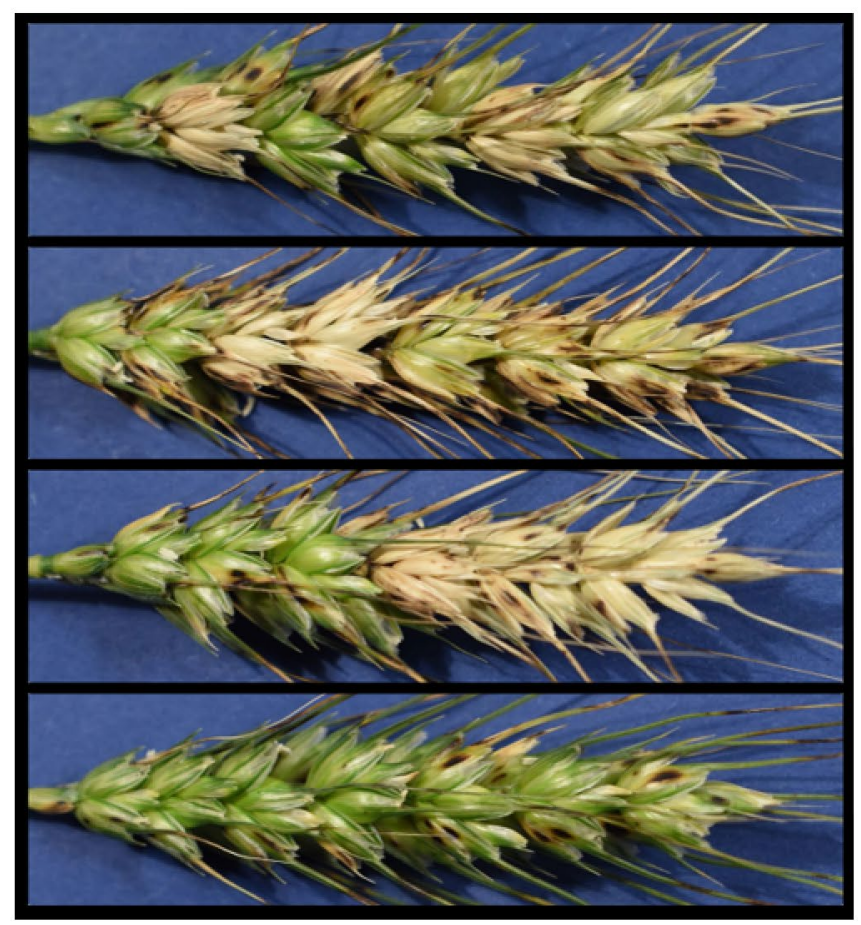

untreated control

seed treatment

spray treatment

on flag leaf, $7 \mathrm{~d}$ prior

to inoculation

drench treatment at

stage of flag leaf

expansion $7 \mathrm{~d}$ prior

to inoculation

Fig. 4 Spike infection with the wheat blast fungus $c v$. Apogee. The effectiveness of isotianil in controlling wheat blast on spikes was evaluated in response to three different types of treatment, i.e. seed, drench and spray application, respectively. While seed coating (50 g a.i./dt) was done prior to sowing, drenching (26 mg a.i./pot) and spraying (200 g a.i./ha) took place on the fully emerged flag leaves. One week after these treatments, spikes had developed and were inoculated with the virulent isolate BR 32. Pictures of spikes were taken 17 days after inoculation 
in wheat were described as being induced after treatment with benzothiadiazole (BTH) and named 'WHEAT CHEMICALLY INDUCED' (WCI) genes (Gorlach et al. 1996). Here, we tested whether the wheat gene WCI-2, encoding a lipoxygenase, also responds to isotianil treatment. Therefore, wheat plants were treated with isotianil by drenching at the stage of fully expanded primary leaves (around 7 days after sowing). Then, quantitative real-time (qRT) PCR was used to monitor WCI-2 transcript abundance in the newly emerged second leaves. This revealed a more than ten times increase in transcript abundance at the fourth day after treatment (Fig. 5a). Next, treatment with isotianil was combined with pathogen inoculation. In this assay, plants were first treated with isotianil on the first emerged leaf and then inoculated with a compatible MoT isolate after emergence of the second leaf
(5 days after isotianil treatment). RNA was extracted from the inoculated leaf, and qRT-PCR analysis again confirmed a significant accumulation of $\mathrm{WCI}-2$ transcripts in response to isotianil. However, pathogen inoculation alone did not lead to an increase in WCI-2 transcripts and combining isotianil treatment with inoculation also showed no enhancement of the isotianil-dependent increase (Fig. 5b).

\section{Discussion}

Wheat blast emerged as novel threat to wheat cultivation and has the potential to tremendously impact global food security. One key question in the debate of how to fight
Fig. 5 Relative transcript abundance of WCI-2 after isotianil treatment. Plants of wheat $c v$. Little Club were treated with isotianil 7 days after sowing at the stage of primary leaf expansion. a Treatment was done by drenching each pot with $26 \mathrm{mg}$ a.i. The second leaf from nine individual plants was harvested at time points indicated and used for RNA isolation. b Plants were drenched with isotianil at $13 \mathrm{mg}$ a.i./pot, and 5 days after treatment (120 hpt), all plants were inoculated on the second leaf with the compatible MoT isolate BR32. RNA was isolated from these secondary leaves at different time points after inoculation. RNA from a and $\mathbf{b}$ was transcribed to cDNA and used for quantification of WCI-2 transcript abundance by RT-qPCR. The expression of WCI-2 was calculated relative to EF1 $\alpha$. Values shown represent the mean \pm standard deviation of three samples consisting of two leaves each. Results shown are from a single experiment. hpt, hours post-treatment; hpi, hours post-inoculation

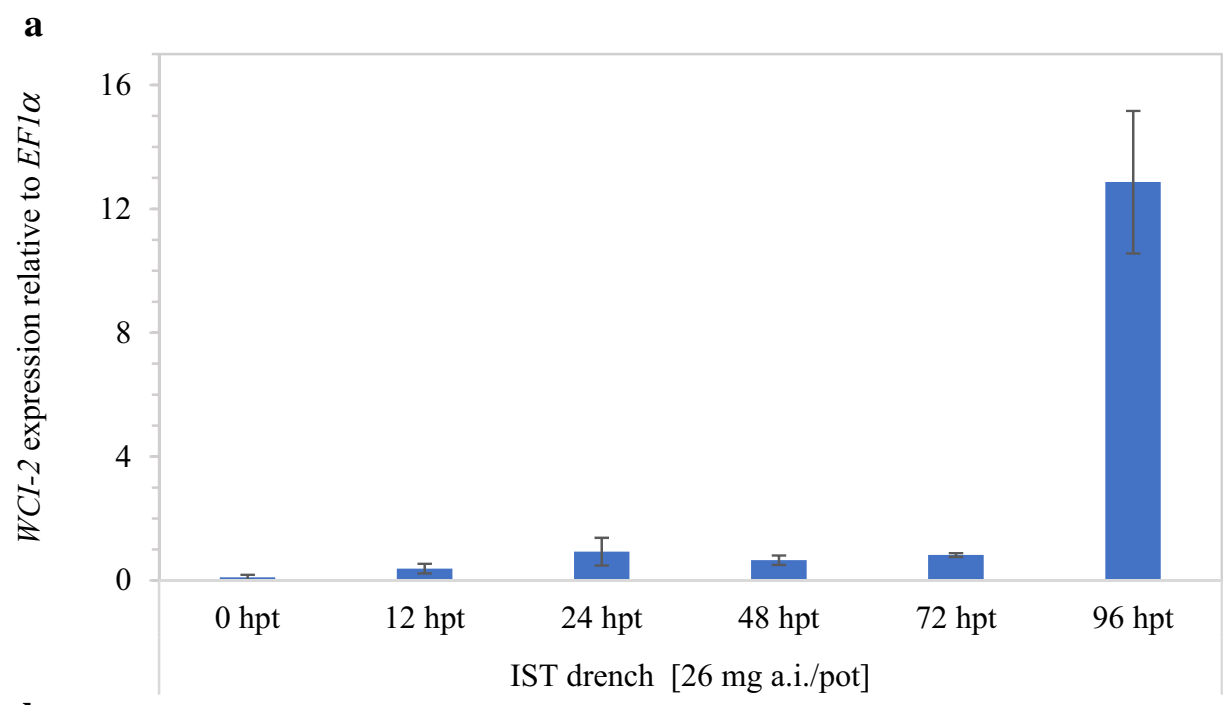

b

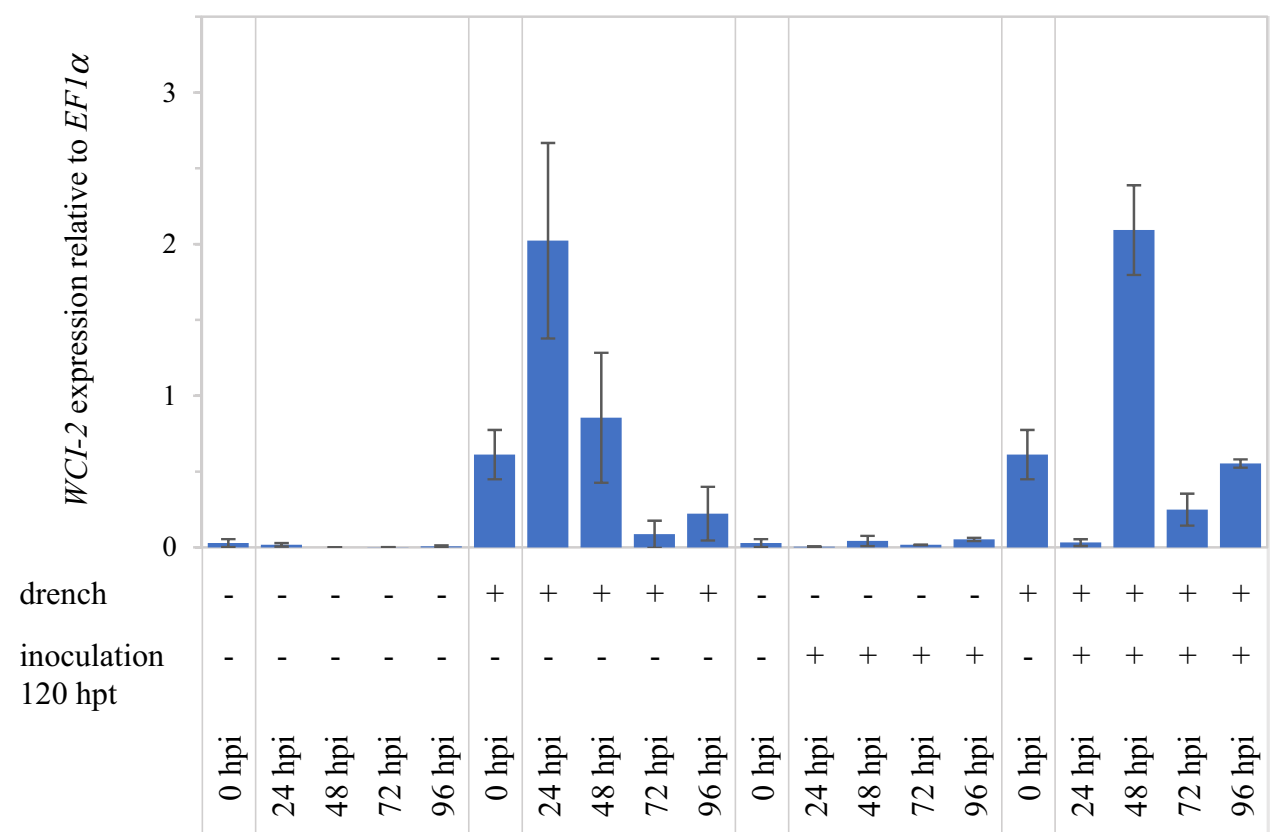


against this disease is whether our extensive knowledge on blast disease on rice, caused by Magnaporthe oryzae, can be used as blueprint for the investigation of wheat blast. Here, we present a study on the use of isotianil, a well-known plant protection against rice blast, to control wheat blast. Our results demonstrated that isotianil is similarly effective in wheat as it is in rice.

Isotianil was launched in 2010 in Japan and Korea as a protection against rice blast and bacterial leaf blight (Toquin et al. 2012). Due to the specific requirements of rice cultivation, as a submerged cropping system, the product was optimized either for seed coating or drench application in seed boxes. Accordingly, these two application methods were first evaluated in wheat and were found to provide a highly significant level of protection against wheat blast at the seedling stage (Fig. 1, Online Resources 1 and 2). Doses of $10 \mathrm{mg}$ a.i. per dt seeds and $13 \mathrm{mg}$ a.i. per pot were shown to be sufficient for seed and drench application, respectively, to gain a 70 to $95 \%$ reduction in diseased leaf area (Fig. 1). These doses are of agronomical relevance and similar to those used in rice cultivation (Toquin et al. 2012). Because in wheat cropping systems, fungicides are mostly applied by spraying, we additionally evaluated this method of treatment. Spray application, however, turned out to be less effective compared to the aforementioned treatments (Fig. 1 and Online Resource 3). This could either mean that the active ingredient is not sufficiently taken up by wheat leaves, potentially due to an inappropriate formulation for the leaf type, or that isotianil is not properly transported within the plant, i.e. first by basipetal and then an acropetal transport mechanism. Nonetheless, results after seed and drench application confirmed the efficacy of isotianil against $M o T$ in wheat. Remarkably, this holds true even at different disease pressures as exemplified by $1-55 \%$ and 6-70\% diseased leaf area in the untreated control of experiments with seed and drench treatment, respectively (Online Resources 1 and 2). To further substantiate this observation, we tested the protection provided by drenching with isotianil against MoT isolates of different origins. Even against the most aggressive isolates, AR33 and AR06, the reduction in disease severity was similarly high as compared with the less aggressive isolates, BR32 and BR116.5 (Fig. 2, Online Resource 4). These results underpin that synthetic plant defence inducers, such as isotianil, are even effective at low and high disease pressure. Using quantitative microscopy, it could be demonstrated that the treatment with isotianil led to an inhibition of invasive growth of the pathogen, i.e. colonization was stopped during transition from epidermal to mesophyll tissue (Fig. 3). To further rule out the possibility that the $M o T$ isolates used in this study are less vital, possibly due to the presence of a mutation conferring resistance to strobilurin fungicides (Hawkins and Fraaije 2018), a PCR-based approach was undertaken. Using specific primers for amplification of gene fragments spanning the respective regions, it was shown that neither the G143A nor the F129L amino acid swap was present in any of the isolates (Online Resource 5). These mutations are already quite common in $M o T$ populations in Brazil, and these already limit the use of classical fungicides and highlight the necessity for alternatives such as isotianil (Castroagudin et al. 2015).

The most severe and even most apparent indication for wheat blast disease in fields are spike infections which, in extreme cases, can lead to complete bleaching of a spike (Cruz and Valent 2017). Because seedling resistance on leaves and adult plant resistance on spikes are not strictly correlated (Martínez et al. 2019), an investigation of both plant stages and organs is necessary. Under laboratory conditions, experiments with wheat spikes are difficult to conduct because of the long time needed between sowing and flowering and the growing space needed for large wheat plants at the adult stage. In this respect, a particularly useful experimental tool is the spring wheat cultivar 'Apogee' which has an exceptionally short life cycle and flowers 25 days after planting, without vernalization ( $\mathrm{Li}$ et al. 2017). First, it was confirmed that $c v$. Apogee was similarly protected by isotianil, against wheat blast at the seedling stage, as was demonstrated for $c v$. Little Club (Fig. 2, Online Resource 4). In wheat cropping, a final fungicide treatment is routinely applied at the flag leaf stage. Therefore, $c v$. Apogee plants were treated at this stage by drench and spray application with isotianil and compared to plants emerged from seeds coated with isotianil. The evaluation of disease symptoms on spikes around 3 weeks after inoculation revealed that drench application was the most effective treatment (Fig. 4). Monitoring the amount of shriveled seeds per spike gave further support to this observation (Online Resource 7a) linking reduced spike infections to increased yield.

Interestingly, the combination of seed treatment followed by drench application most efficiently reduced the proportion of malformed seeds (Online Resource 7b). This observation might pave the way for the use of isotianil in wheat cropping because it provides a long period of protection from newly emerged seedling to mature plant prior to harvest. It was shown that transcripts of the wheat gene WCI-2 strongly accumulate after isotianil treatment (Fig. 5). This suggests that isotianil functions as a defence inducer in wheat, as previously shown for BTH (Gorlach et al. 1996). Additionally, it was observed that other diseases of wheat, i.e. powdery mildew, could also be controlled by isotianil (Online Resource 8) which confirmed previously published data (Toquin et al. 2012).

In summary, the presented data have shown isotianil as an interesting new tool for the management of wheat blast disease. 
Acknowledgements The authors are thankful to Prof. Analia Perello from University La Plata, Argentina, for helpful discussions. Dr. Richard Meredith from Bayer AG is kindly acknowledged for proof reading of the manuscript and helpful discussion.

Author contribution $\mathrm{KP}, \mathrm{FC}$ and $\mathrm{AJ}$ performed experiments and evaluated results. SB, DP and AM were involved in the experimental design and helped in finalizing the manuscript. US designed the study, accompanied the experiments, drafted and finalized the manuscript.

Funding Open Access funding enabled and organized by Projekt DEAL.

\section{Compliance with ethical standards}

Conflict of interest This work was funded in part by Bayer AG, Division CropScience. DP and AM are employees of Bayer AG.

Human and animal rights The authors did not perform any study with animals or humans' participants to produce this manuscript.

Open Access This article is licensed under a Creative Commons Attribution 4.0 International License, which permits use, sharing, adaptation, distribution and reproduction in any medium or format, as long as you give appropriate credit to the original author(s) and the source, provide a link to the Creative Commons licence, and indicate if changes were made. The images or other third party material in this article are included in the article's Creative Commons licence, unless indicated otherwise in a credit line to the material. If material is not included in the article's Creative Commons licence and your intended use is not permitted by statutory regulation or exceeds the permitted use, you will need to obtain permission directly from the copyright holder. To view a copy of this licence, visit http://creativecommons.org/licenses/by/4.0/.

\section{References}

Bektas Y, Eulgem T (2015) Synthetic plant defense elicitors. Front Plant Sci 5:804. https://doi.org/10.3389/fpls.2014.00804

Castroagudin VL, Ceresini PC, de Oliveira SC, Reges JT, Maciel JL, Bonato AL et al (2015) Resistance to QoI fungicides is widespread in Brazilian populations of the wheat blast pathogen Magnaporthe oryzae. Phytopathology 105(3):284-294. https://doi. org/10.1094/PHYTO-06-14-0184-R

Castroagudín VL, Moreira SI, Pereira DAS, Moreira SS, Brunner PC, Maciel JLN et al (2016) Pyricularia graminis-tritici, a new Pyricularia species causing wheat blast. Persoonia 37:199-216. https://doi.org/10.3767/003158516X692149

Ceresini PC, Castroagudín VL, Rodrigues FÁ, Rios JA, Aucique-Pérez CE, Moreira SI et al (2019) Wheat blast: from its origins in South America to its emergence as a global threat. Mol Plant Pathol 20(2):155-172. https://doi.org/10.1111/mpp.12747

Conrath U (2009) Priming of induced plant defense responses. Adv Bot Res 51:361-395

Cruz CD, Valent B (2017) Wheat blast disease: danger on the move. Trop Plant Pathol 42(3):210-222. https://doi.org/10.1007/s4085 8-017-0159-z

Delventhal R, Falter C, Strugala R, Zellerhoff N, Schaffrath U (2014) Ectoparasitic growth of Magnaporthe on barley triggers expression of the putative barley wax biosynthesis gene CYP96B22 which is involved in penetration resistance. BMC Plant Biol 14(1):26. https://doi.org/10.1186/1471-2229-14-26

Ellis JG, Lagudah ES, Spielmeyer W, Dodds PN (2014) The past, present and future of breeding rust resistant wheat. Front Plant Sci. https://doi.org/10.3389/fpls.2014.00641

Gladieux P, Condon B, Ravel S, Soanes D, Maciel JLN, Nhani A et al (2018) Gene flow between divergent cereal- and grass-specific lineages of the rice blast fungus Magnaporthe oryzae. mBio 9(1):e01217-e01219. https://doi.org/10.1128/mbio.01219-17

Gorlach J, Volrath S, Knauf-Beiter G, Hengy G, Beckhove U, Kogel $\mathrm{KH}$ et al (1996) Benzothiadiazole, a novel class of inducers of systemic acquired resistance, activates gene expression and disease resistance in wheat. Plant Cell 8(4):629-643. https://doi. org/10.1105/tpc.8.4.629

Hawkins NJ, Fraaije BA (2018) Fitness penalties in the evolution of fungicide resistance. Annu Rev Phytopathol 56(1):339-360. https ://doi.org/10.1146/annurev-phyto-080417-050012

Inoue Y, Vy TTP, Yoshida K, Asano H, Mitsuoka C, Asuke S et al (2017) Evolution of the wheat blast fungus through functional losses in a host specificity determinant. Science 357(6346):80-83. https://doi.org/10.1126/science.aam9654

Islam MT, Croll D, Gladieux P, Soanes DM, Persoons A, Bhattacharjee $P$ et al (2016) Emergence of wheat blast in Bangladesh was caused by a South American lineage of Magnaporthe oryzae. BMC Biol 14(1):84. https://doi.org/10.1186/s12915-016-0309-7

Jarosch B, Jansen M, Schaffrath U (2003) Acquired resistance functions in mlo barley, which is hypersusceptible to Magnaporthe grisea. Mol Plant Microbe Interact 16(2):107-114

Jarosch B, Collins NC, Zellerhoff N, Schaffrath U (2005) RAR1, ROR1, and the actin cytoskeleton contribute to basal resistance to Magnaporthe grisea in barley. Mol Plant Microbe Interact 18(5):397-404

Jeschke P (2016) Progress of modern agricultural chemistry and future prospects. Pest Manag Sci 72(3):433-455. https://doi.org/10.1002/ ps. 4190

Li G, Boontung R, Powers C, Belamkar V, Huang T, Miao F et al (2017) Genetic basis of the very short life cycle of 'Apogee' wheat. BMC Genom 18(1):838. https://doi.org/10.1186/s1286 4-017-4239-8

Livak KJ, Schmittgen TD (2001) Analysis of relative gene expression data using real-time quantitative PCR and the 2(-Delta Delta C(T)) method. Methods 25(4):402-408. https://doi.org/10.1006/ meth.2001.1262

Ma B, Uddin W (2009) Fitness and competitive ability of an Azoxystrobin-resistant G143A mutant of Magnaporthe oryzae from perennial ryegrass. Plant Dis 93(10):1044-1049. https://doi. org/10.1094/pdis-93-10-1044

Martínez SI, Sanabria A, Fleitas MC, Consolo VF, Perelló A (2019) Wheat blast: aggressiveness of isolates of Pyricularia oryzae and effect on grain quality. J King Saud Univ Sci 31(2):150-157. https ://doi.org/10.1016/j.jksus.2018.05.003

McGrann GRD, Steed A, Burt C, Nicholson P, Brown JKM (2015) Differential effects of lesion mimic mutants in barley on disease development by facultative pathogens. J Exp Bot 66(11):34173428. https://doi.org/10.1093/jxb/erv154

Mogga V, Delventhal R, Weidenbach D, Langer S, Bertram PM, Andresen K et al (2016) Magnaporthe oryzae effectors MoHEG13 and MoHEG16 interfere with host infection and MoHEG13 counteracts cell death caused by Magnaporthe-NLPs in tobacco. Plant Cell Rep 35(5):1169-1185. https://doi.org/10.1007/s0029 9-016-1943-9

Olivera Firpo PD, Newcomb M, Flath K, Sommerfeldt-Impe N, Szabo LJ, Carter M et al (2017) Characterization of Puccinia graminis f. sp. tritici isolates derived from an unusual wheat stem rust outbreak in Germany in 2013. Plant Pathol 66(8):1258-1266. https ://doi.org/10.1111/ppa.12674 
Pennisi E (2010) Armed and dangerous. Science 327(5967):804-805. https://doi.org/10.1126/science.327.5967.804

Rajaram S (2011) Norman Borlaug: the man i worked with and knew. Annu Rev Phytopathol 49(1):17-30. https://doi.org/10.1146/annur ev-phyto-072910-095308

Sadat MA, Choi J (2017) Wheat blast: a new fungal inhabitant to Bangladesh threatening world wheat production. Plant Pathol J 33(2):103-108. https://doi.org/10.5423/PPJ.RW.09.2016.0179

Sardesai N, Subramanyam S, Nemacheck J, Williams CE (2005) Modulation of defense-response gene expression in wheat during Hessian fly larval feeding. J Plant Interact 1(1):39-50. https://doi. org/10.1080/17429140500309498

Saunders DGO, Pretorius ZA, Hovmøller MS (2019) Tackling the reemergence of wheat stem rust in Western Europe. Commun Biol 2(1):51. https://doi.org/10.1038/s42003-019-0294-9

Strugala R, Delventhal R, Schaffrath U (2015) An organ-specific view on non-host resistance. Front Plant Sci. https://doi.org/10.3389/ fpls.2015.00526

Toquin V, Sirven C, Assmann L, Sawada H (2012) Host Defense inducers. In: Krämer W, Schirmer U, Jeschke P, Witschel M (eds) Modern crop protection compounds, John Wiley and Sons. https://doi. org/10.1002/9783527644179.ch26
Valent B, Farman M, Tosa Y, Begerow D, Fournier E, Gladieux P et al (2019) Pyricularia graminis-tritici is not the correct species name for the wheat blast fungus: response to Ceresini et al. (MPP 20:2). Mol Plant Pathol 20(2):173-179. https://doi.org/10.1111/ mpp. 12778

Wunderle J, Leclerque A, Schaffrath U, Slusarenko A, Koch E (2012) Assessment of the loose smut fungi (Ustilago nuda and $U$. tritici) in tissues of barley and wheat by fluorescence microscopy and real-time PCR. Eur J Plant Pathol 133(4):865-875. https://doi. org/10.1007/s10658-012-0010-9

Zhang H, Zheng X, Zhang Z (2016) The Magnaporthe grisea species complex and plant pathogenesis. Mol Plant Pathol 17(6):796-804. https://doi.org/10.1111/mpp.12342

Publisher's Note Springer Nature remains neutral with regard to jurisdictional claims in published maps and institutional affiliations. 